\title{
Impact of particle-size reduction on solubility and antidiabetic activity of extracts of leaves of Vinca rosea
}

\author{
Khalid Hussain, Abida Qamar, Nadeem Irfan Bukhari, Amjad Hussain, Naureen Shehzadi, Shaista \\ Qamar, Sajida Parveen \\ Punjab University College of Pharmacy, University of the Punjab, Lahore-54000, Pakistan
}

\section{ABSTRACT}

Introduction: The present study aimed to enhance aqueous-solubility of methanol extract of leaves of Vinca rosea (Family: Apocynaceae) by particle-size reduction using milling, and evaluate its antidiabetic activity.

Methods: The methanol extract (ME) was micronized using Vibratory Ball Mill, operated at vibratory speed of $15 \mathrm{~Hz}$ for 60 min, room temperature, and the resulting extract (MME) was investigated to determine particle-size, solubility, UV/Visible profile and in vitro antidiabetic activity.

Results: The average particle-size of MME was found to be $0.753 \pm 0.227 \mu \mathrm{m}$ which was less than half of the ME $(2.007 \pm 0.965 \mu \mathrm{m})$. The solubility of MME was found to be greater than the ME. MME exhibited $65.63,18.0$ and $96.87 \%$ higher antidiabetic activity in the glucose uptake by yeast cells method, hemoglobin glycosylation assay and the alpha amylase inhibition assay, respectively $(p<0.05)$.

Discussion and conclusion: The results of the present study indicate that micronization has effectively enhanced the aqueous solubility and antidiabetic activity of methanol extract of leaves of Vinca rosea.

Keywords: Vinca rosea, Methanol extract, Micronization, Solubility, Antidiabetic activity INTRODUCTION 
The surge in interest of masses towards plant-based drugs is increasing day-by-day. But, mostly such products show poor aqueous-solubility and oral bioavailability leading challenges in formulation development and efficacy. As a result, active constituents cannot reach the target site at a rate and extent, needed to show/elicit therapeutic response. Several techniques may be used to improve aqueous-solubility and oral bioavailability. Among such techniques, particle-size-reduction (micronization) is one of the oldest approaches to improving solubility. ${ }^{1}$ Micronization, a conventional technique for size reduction, is commonly used for enhancing solubility. This technique reduces particle-size up to $2-5 \mu \mathrm{m}$ usually, but sometime below $1 \mu \mathrm{m} .{ }^{2}$ For such purpose, the Jet Milling, Ball Milling and High Pressure Homogenization Machines are being used frequently. ${ }^{1}$ In the present study, the Ball Mill was used to reduce the particle-size of extract of leaves of a traditional medicinal plant, Vinca rosea.

The plant is well-known due to its alkaloids such as vincristine and vinblastine for treating cancer. ${ }^{3} \mathrm{As}$ a folklore medicine, fresh leaves of the plant are chewed to manage diabetes. The scientific evidence of this use was reported due to isolation of alkaloids such as vindoline, vindolidine, vindolicine and vindolinine which had antidiabetic activity. ${ }^{4}$ The three alkaloids exhibited quite high median inhibitory concentration for cell viability, hence considered safe to consume. Moreover, such alkaloids are poorly soluble in aqueous medium and suspected to have low systemic level so that to exhibit cytotoxicity. The extracts of the plant contains flavonoids and polyphenols which have antidiabetic activity too. ${ }^{5}$ The activity of extract of the plant may be enhanced by increasing aqueous-solubility by micronization. Therefore, the present study aimed to reduce the particle-size of methanol extract (ME) of leaves of Vinca rosea and evaluate antidiabetic activity of micronized methanol extract (MME). The results of the present study may enhance the utilization of this plant for treating/managing diabetes. 


\section{MATERIALS AND METHODS}

\section{Collection and extraction}

The plant was acquired from the National Agricultural Research Centre, Islamabad, Pakistan. The leaves were separated, washed, dried under shade and pulverized. Powdered material $(75 \mathrm{~g})$ was macerated with $200 \mathrm{~mL}$ methanol for five days. The solvent was removed and the extraction was repeated thrice using the same volume of methanol. The extract was filtered and dried in vacuo at $40^{\circ} \mathrm{C}$, and termed as ME.

\section{Micronization of extract}

The Vibratory Ball Mill (Locally fabricated, Lahore, Pakistan) was used in this study. It was fitted with two stainless steel cylinders (10 cm length, $32 \mathrm{~mm}$ internal diameter) and each containing one stainless steel ball ( $25 \mathrm{~mm}$ diameter). A total of $3 \mathrm{~g} \mathrm{ME}$ was added in to each of the cylinders, fitted in the mill and micronized by operating the machine at vibratory speed of $15 \mathrm{~Hz}$ for $60 \mathrm{~min}$ at room temperature. The resulting extract was termed as MME.

\section{Chemicals}

Metronidazol (Siza International, Lahore, Pakistan), alpha amylase and hemoglobin (China), acarbose (Bayer, Pakistan), methanol (RCl Labscan, Thailand), glucose, starch, gallic acid and enthrone reagent (Sigma Aldrich) were procured from the local market.

\section{Characterization of ME and MME}

$M E$ and MME were subjected to scanning electron microscopy(SEM) to determine the particle morphology in the magnification range from lower to higher (1.00 kx and 25.0 kx). The various sized particles were observed in magnification range of $10.0 \mathrm{kx}$ and average particle-size was determined by image $\mathrm{J}$ Tool (software). Both the extracts were dissolved in methanol to get the solutions having a final concentration of 1.0 $\mathrm{mg} / \mathrm{mL}$. These solutions were scanned in UV/Visible range $(800-200 \mathrm{~nm})$ and the 
spectra were compared with each other. The aqueous solubility of ME and MME was assessed by taking $10 \mathrm{mg}$ extract in separate test tubes containing $10 \mathrm{~mL}$ water. The samples were allowed to dissolve by shaking with hands and if not soluble subjected to sonication for $1 \mathrm{~min}$. The formation of clear homogenous solution indicated the solubility.

\section{Antidiabetic activity}

\section{Glucose uptake by yeast cells}

The method described by Kumar et al. was used to study glucose uptake by yeast cells. ${ }^{6}$ Briefly, the yeast cells were rinsed with distilled water by centrifugation at 2500 rpm for $5 \mathrm{~min}$ and the procedure was repeated until supernatant became clear. Then, yeast cell pellet was suspended in water to prepare $10 \%$ suspension (v/v). One milliliter of $10 \mathrm{mM}$ glucose solution and $1 \mathrm{~mL}$ of each extract/standard (metronidazole) were mixed and incubated at $37^{\circ} \mathrm{C}$ for $10 \mathrm{~min}$. Then, $100 \mu \mathrm{L}$ yeast suspension was added mixed and incubation was continued for $1 \mathrm{~h}$ at $37^{\circ} \mathrm{C}$. Afterwards, the mixture was centrifuged at $2500 \mathrm{rpm}$ for $5 \mathrm{~min}$ and the supernatant was used to determine the glucose concentration. The percentage glucose uptake was computed by using the following formula:

$$
\text { Percentage uptake }=-\frac{[\text { Absorbance of control }- \text { Absorbance of sample }]}{\text { Absorbance of control }}
$$

\section{Hemoglobin glycosylation inhibition activity}

The activity was determined using the procedure described by Parker et al. ${ }^{7}$ Briefly, 1 $\mathrm{mL}$ of hemoglobin $(0.06 \%, \mathrm{w} / \mathrm{v})$, gentamycin $(0.02 \%, \mathrm{w} / \mathrm{v})$, sample/standard (gallic acid) solution, and glucose $(2 \%, w / v)$ were mixed and incubated in the dark at room temperature for $72 \mathrm{~h}$. Then, the absorbance was measured at $440 \mathrm{~nm}$ and percentage inhibition of hemoglobin glycosylation was determined using the formula given as follows:

$$
\text { Percentage activity }=\frac{[\text { Absorbance of control }- \text { Absorbance of sample }]}{\text { Absorbance of control }}
$$

\section{Alpha amylase inhibition activity}


The activity was determined using the method developed by Ramakrishna et al. ${ }^{8}$ Briefly, $1 \mathrm{~mL}$ of enzyme solution $(0.5 \mathrm{mg} / \mathrm{mL}$, in $20 \mathrm{mM}$ phosphate buffer of $\mathrm{pH} 6.9)$ and $1 \mathrm{~mL}$ of extract/standard (acarbose) solution were mixed and incubated at $37^{\circ} \mathrm{C}$ for $10 \mathrm{~min}$. Then, $1 \mathrm{~mL}$ of $1 \%$ starch solution was added and the reaction mixture was again incubated at $37^{\circ} \mathrm{C}$ for $10 \mathrm{~min}$. Finally, reaction was stopped by adding $2 \mathrm{~mL}$ dinitrosalycic acid and the mixture was further heated in boiling water bath for $8 \mathrm{~min}$. The contents were cooled and the absorbance was measured at $540 \mathrm{~nm}$. The \% inhibition of the enzyme activity was calculated by the formula given as follows

$$
\text { Percentage activity }=\frac{[\text { Absorbance of control }- \text { Absorbance of sample }]}{\text { Absorbance of control }}
$$

\section{Statistical analysis}

The data were analyzed by one way ANOVA with Bonferoni Post Hoc multiple comparison. A P value $<0.05$ was considered a significantly different.

\section{RESULT AND DISCUSSION}

\section{Micronization and characterization of extracts}

The MME and ME of leaves of Vinca rosea were compared with each other in terms of morphology, size distribution, solubility, UV/Visible absorbance behavior and antidiabetic activity. The morphology of ME and MME, determined by SEM in lower and higher magnification (1.0 kx and $25.0 \mathrm{kx}$ ), is shown in the Figure 1 and 2, respectively. The particle-size distribution, determined in the magnification range of $10.0 \mathrm{kx}$, of $\mathrm{ME}$ and $M M E$ is given in Figure 3. The particles of ME appeared angular with low sphericity, whereas particles of MME appeared rounded with medium sphericity. The sphericalshaped smaller particles showed agglomeration due to micronization process. These results show that micronization has enhanced flowability, packing and interaction with fluids and the covering power of pigments, which are the much needed properties of a pharmaceutical material.

The average particle-size of ME prior to micronization, determined from SEM data by applying image $\mathrm{J}$ Tool (software), was found to be $2.007 \pm 0.0965 \mu \mathrm{m}$, whereas the 
average particle-size of MME was found to be $0.753 \pm 0.227 \mu \mathrm{m}(62.48 \%$ reduction in size). Hence, the milling had increased the surface area of the particles.

The impact of micronization on aqueous-solubility of the extract was found to be positive. The solubility ME in distilled water was found to be $2 \mathrm{mg} / \mathrm{mL}$ with sonication, whereas MME was found to be soluble in water in the same proportion without sonication. This ease in solubility was due to smaller particle-size as described by the Noyes Whitney Equation, which indicated that when particles became smaller, the surface area to volume ratio was increased. The larger surface area allowed greater interaction with the solvent molecules which resulted in increased solubility. The reduction in the particle-size increases the rate of solution because of large surface area. ${ }^{9}$

The overlays of UV/Visible spectra of ME and MME of leaves of Vinca rosea is shown in the Figure 4. The spectra of both the extracts were found to be superimposable indicating chemical similarity of both the extracts. These results clearly indicated that milling had not affected the chemical nature of the constituents of the extract. So, the solubility was increased due to milling but without any chemical change.

The improvement in the solubility of MME of leaves of Vinca rosea is of a great importance. The plant is reported to contain antidiabetic alkaloids and polyphenols/flavonoids which are either insoluble or poorly soluble in water. In the present study, plant material was de-fated with petroleum ether and then alkaloidal contents were further reduced by extracting the residue with chloroform in which alkaloids were soluble. The residue was extracted with methanol so that the extract contained polar compounds such as polyphenols and flavonoids in higher proportion. This extract was subjected to milling to enhance aqueous-solubility of MME and its chemical constituents leading to more efficacy. To confirm this fact, both types of extracts were investigated for antidiabetic activity, traditional use of the plant, using different in vitro models.

\section{Antidiabetic activity}


The effect of micronization on antidiabetic activity of ME and MME of leaves of the plant using three in vitro models is shown in the Figure 5. In all the three models, MME had shown higher activity as compared to ME and standard drug $(p<0.05)$. The activity of MME was found to be $63 \%$ higher in the glucose uptake by yeast cell assay, $18.0 \%$ higher inhibition in hemoglobin-glycosylation inhibition assay, and $96.87 \%$ higher in alpha amylase inhibition assay than that of ME. These results explicitly indicate the positive effect of physical modification of particle-size on hypoglycemic activity.

In the present study, MME has shown higher uptake of glucose in yeast cells as compared to ME. This increase in activity is due to reduction in particle-size and higher aqueous solubility. Owing to such behavior the constituents of the extract can enter in yeast cells at a higher rate and extent, thereby facilitating glucose utilization within the cell. This creates a concentration gradient across the membrane and facilitates the movement of glucose from the solution to the cell. It is reported that glucose uptake in the yeast cells takes place through facilitated diffusion which is catalyzed by glucose transporter and hence the compounds enhancing the activity of the transporter can increase the glucose uptake. ${ }^{10}$ As the glucose enters in the yeast cell, phosphorylation takes place which prevents the glucose molecules to diffuse back and create a concentration gradient. ${ }^{10}$ Therefore, MME entered the cells much faster than ME and enhanced the transport of glucose from the solution to the cells due to facilitated diffusion and phosphorylation

Likewise, the inhibition of $\mathrm{Hb}$ glycosylation with MME is higher as compared to ME. The gycosylation can be inhibited by two ways; blocking the glucose so that it cannot interact with amino group of the beta chain of hemoglobin, and blocking the amino group of the hemoglobin. The activity of the extract may be multifaceted because it contains alkaloids, soluble proteins, polyphenols and flavonoids. The nitrogen groups of the alkaloids and soluble proteins may block aldehyde group of glucose, thus reducing its availability to react with hemoglobin. The polyphenols and flavonoidscan act as proton donors to reduce the aldehyde group of the glucose. The same is reported about 
the glycosylation inhibition activity of polyphenols and flavonoids. ${ }^{11,12}$ The extract might have shown all such effects in glycosylation inhibition.

$\mathrm{Hb}$ glycosylation has attained much importance in the modern world due to its use as a scale in the long-term control of diabetes. The glycosylation is a non-enzymatic process of attaching glucose molecules with amino group of $\mathrm{Hb}$ leading to the formation of advanced glycated end products. Antioxidants prevent this oxidation process and so in a way inhibit the $\mathrm{Hb}$ glycosylation. ${ }^{12}$

The alpha amylase, an important therapeutic target for antidiabetic drugs, inhibitory response of MME is also superior. This enzyme hydrolyses the alpha glycosidic bond and converts starch into glucose. The phenolic compounds of the extract can show alpha amylase inhibition action as reported earlier. ${ }^{13}$ The inhibitory potential of these metabolites is related to the presence of hydroxyl group which form hydrogen-bonding between hydroxyl group and catalytic residue of the binding site of enzyme. ${ }^{13}$ The difference of activity of ME and MME was due to micronization process. The extracts due to differential solubility interacted with the enzyme differently. The micronization process converted the extract into amorphous particles which resulted in improved solubility. The amorphous form is more readily soluble because of higher Gibbs free energy. ${ }^{14}$ The above mentioned results indicated that smaller sized particles with larger surface area might be having improved antidiabetic effect.

Traditional micronization method has some limitations such as morphology, particles properties which are uncontrolled as compare to novel size reduction techniques. Heterogeneous particle shape and agglomeration is observed in ball milling method which can be prevented by the particle engineered techniques. Microinzation using other methods such as The Jet Milling and High Pressure Homogenization can be used to observe the difference between the results.

\section{STUDY LIMITATIONS}


Traditional micronization method has some limitations such as morphology, particles properties which are uncontrolled as compare to novel size reduction techniques. The heterogeneous particle shape and agglomeration is observed in the Ball Milling Method which can be prevented by the particle engineered techniques. Most of the new chemical entities in drug research are poorly water soluble. Therefore, attempt should be made to enhance water solubility by micronization which would in turn increase the pharmacological activity.

\section{CONCLUSION}

The results of the present study conclude that micronization increases the solubility and antidiabetic activity of methanol extract of leaves of Vinca rosea without causing any chemical change.

\section{ACKNOWLEDGEMENT}

We are thankful COMSATS, Lahore Campus, Pakistan for SEM studies.

\section{REFERENCES}

1. Khadka P, Ro J, Kim H, Kim I, Kim JT, Kim H, Cho JM, Yun G, Lee J.Pharmaceutical particle technologies. An approach to improve drug solubility, dissolution and bioavailability. Asian J Pharm Sci. 2014; 9: 304-316.

2.Rawat N, Kumar MS, Mahadevan N. Solubility. Particle-size reduction is a promising approach to improve the bioavailability of lipophillic drugs. Int J Adv Pharm Res.2012;1: 8-18.

3.Farnsworth NR, Bingel AS. Problems and prospects of discovery new drugs from higher plants by pharmacological screening. Springer Verlag, Berlin.1977;1-22.

4.Tiong SH, Looi CY, Hazni H, Arya A, Paydar HJ, Wong WF, Cheah SC, Mustafa MR, Awang K. Antidiabetic and antioxidant properties of alkaloids from Catharanthus roseus (L.) G. Don. Molecules. 2013;18: 9770-9784.

5. Umeno A, Horie M, Murotomi K, Nakajima Y and Yoshida Y. Antioxidative and antidiabetic effects of natural polyphenols and isoflavones. Molecules. 2016; 21: 708.

6. Kumar B, Dinesh A, Mitra M. In vitro and in vivo studies of antidiabetic Indian medicinal plants. A review. J Herb Med Tox. 2009; 3: 9-14.

7. Parker KL, England JD, Da Costa J, Hess RL, Goldstein DE. Improved 
colorimetric essay for glycosylated haemoglobin. Clin Chem.1981; 25: 669-672.

8. Ramakrishna SV, Suseela T, Ghilyan NP, Jalil A, Prema P, Lonsane BK,Ahmed SY.Recovery of amyloglucosidase from moulay bran. Indian J Technol. 1982; 20: 476480.

9.Chaudhary A, Nagaich U, Gulati N, Sharma VK, Khosa RL. Enhancement of solubilization and bioavailability of poorly soluble drugs by physical and chemical modifications: A recent review.J Adv Pharm Edu Res. 2012; 2: 32-67.

10.Weusthuis RA, Pronk JT, Broek PJA, Dijken JPV. Chemostat cultivation as a tool for studies on sugar transport in yeasts. Microbiol Rev. 1994; 616-630.

11.Yeh WJ, Hsia SM, Lee WH, Wu CH. Polyphenols with antiglycation activity and mechanisms of action: A review of recent findings. J Food Drug. 2017; 2 5:8 4 -9 2.

12.Sadowska BI, Galiniak SBG. Kinetics of glycoxidation of bovine serum albumin by glucose, fructose and ribose and its prevention by food components. Molecules. 2014; 19:18828-49.

13.Sales PMD, Souza PMD, Simeoni $\llcorner A$ Magaihaes PDO, Silveira D. a-amylase inhibitors. A review of raw material and isolated compounds from plant source. J Pharm Pharmaceut Sci. 2012;15: 141-183.

14.Graeser K A, Patterson JE, Zeitler JA. The role of configurational entropy in amorphous systems. Pharmaceutics. 2010; 2: 224-244. 

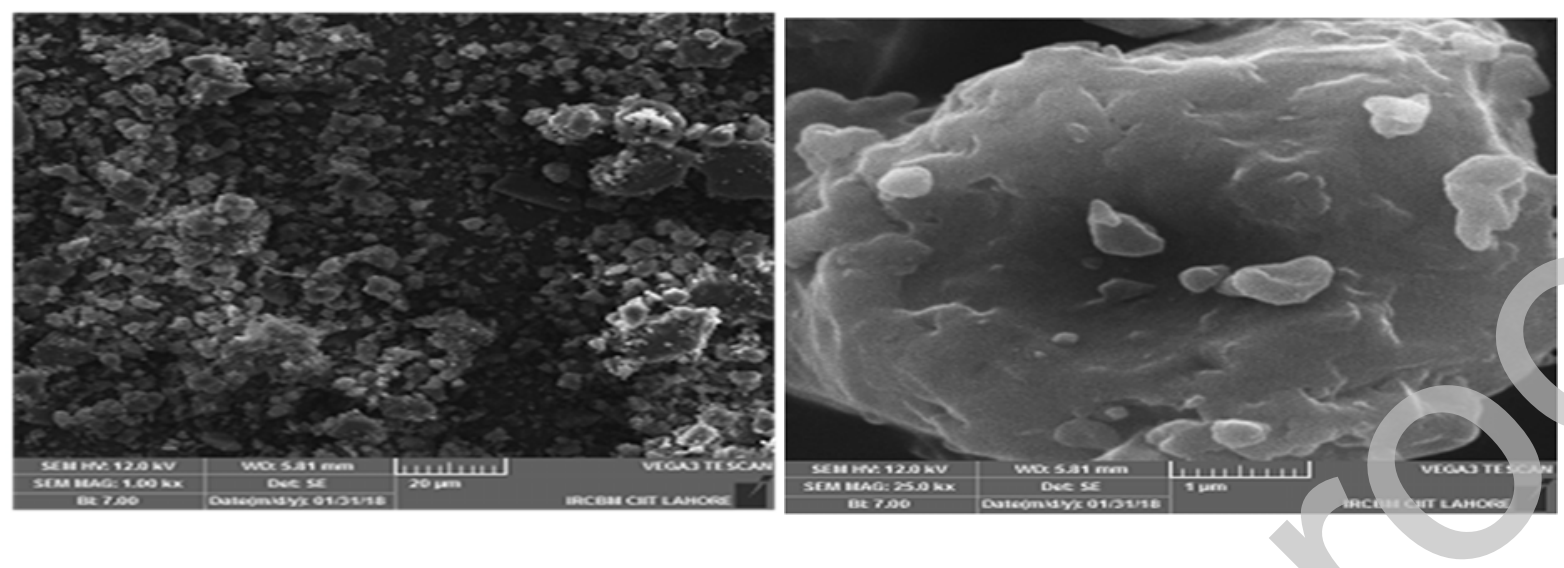

$\mathbf{A}$

B

Figure 1. Scanning electron microscopy (SEM) images of methanol extract of leaves of Vinca rosea in lower $(A)$ and higher magnification (B)

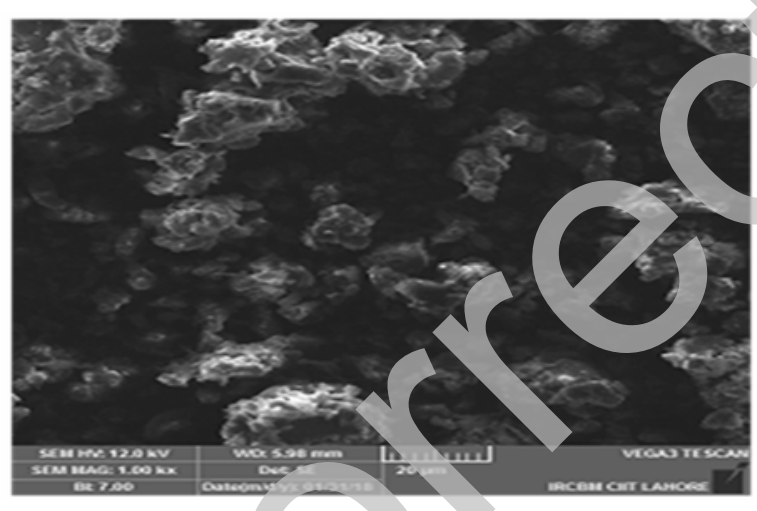

A

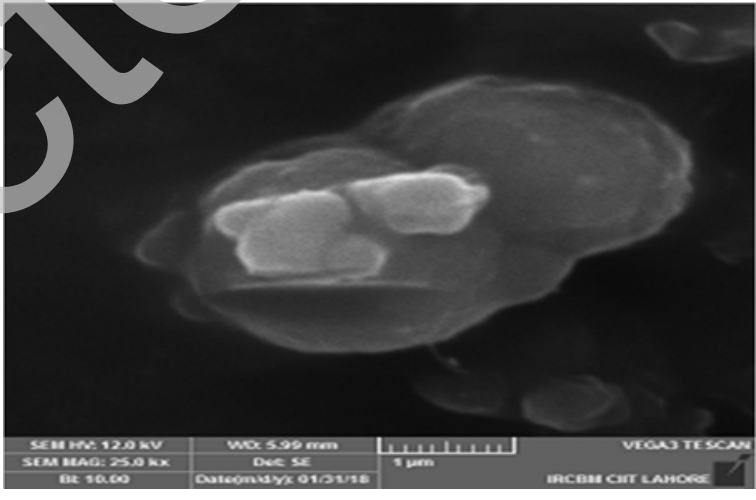

B

Figure 2. Scanning electron microscopy (SEM) images of micronized methanol extract of leaves of Vinca rosea in lower $(A)$ and higher magnification (B) 


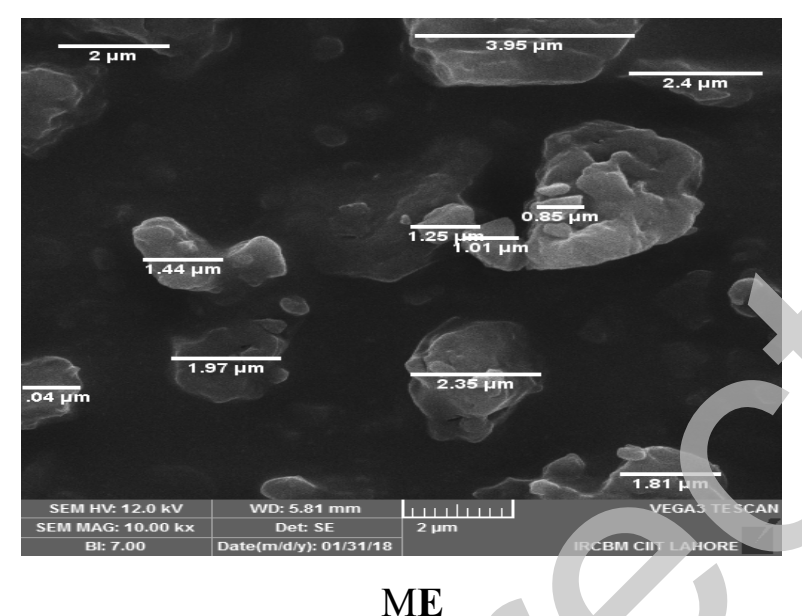

ME

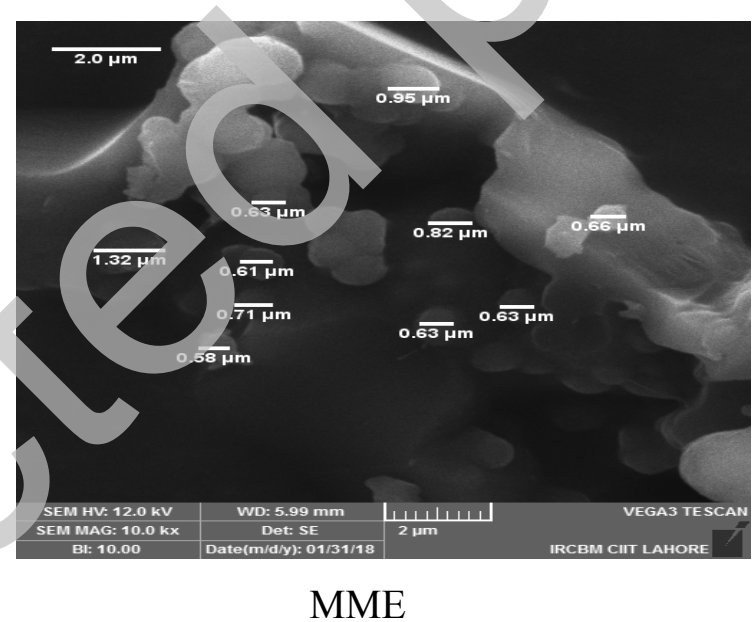

Figure 3. Scanning electron microscopy (SEM) images of methanol (ME) and micronized methanol (MME) extracts showing particles of various sizes in magnification rang of $10.0 \mathrm{kx}$ 


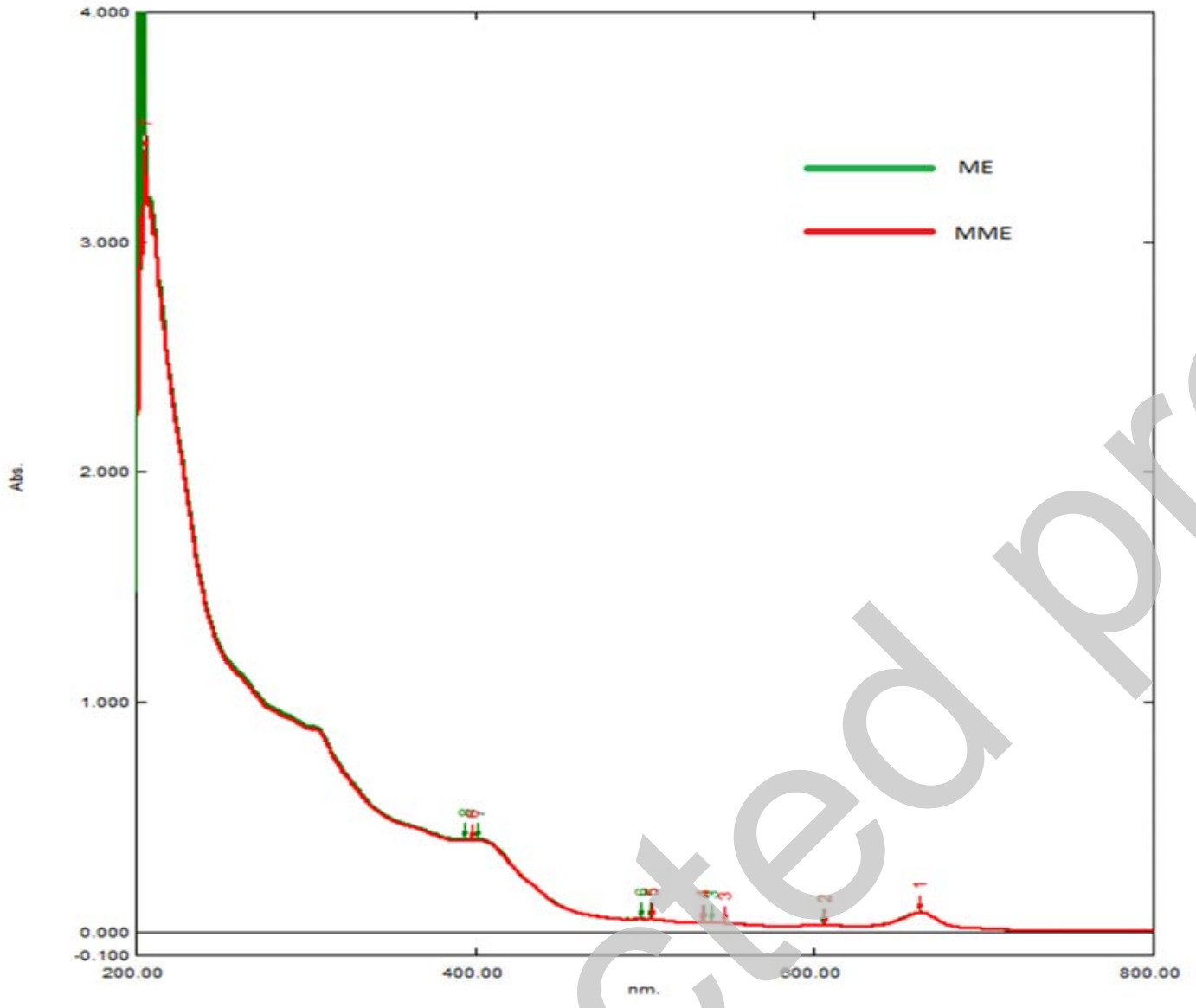

Figure 4.Overlays of UV/Visible profiles of methanol extract (ME) and micronized methanol extract (MME) of leaves of Vinca rosea 


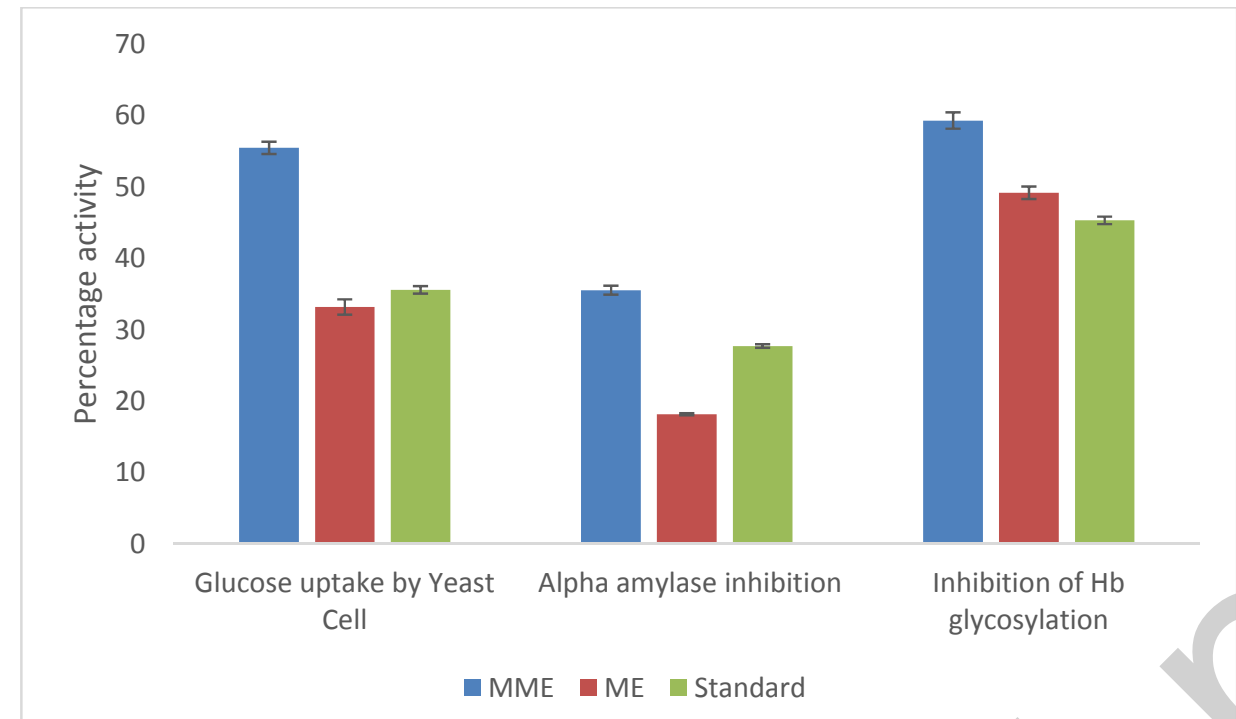

Figure 5. Antidiabetic activity of MME, ME and standard using glucose uptake by yeast cells, Haemoglobin glycosylation and Alpha amylase inhibition assay 